Background As a small hospice, whose only research activity had been individual staff undertaking Masters degrees or local university collaborative service evaluation, opportunity of a national multicentre trial of short specialist palliative care intervention (SSPCI) in advanced neurological disease (AND) was exciting and daunting. The local neurological centre identified suitable patients, explained, consented, randomised and undertook research questionnaires. The hospice delivered intervention, following required training for involved staff.

Method Collation staff/patient comments about study involvement.

Results Staff reported pride at being involved with high calibre institutions; anxious and keen to get it right; initially saw trial patients as different and lacked confidence assessing and evaluating their care; positive experience following patient (with long standing diagnoses, and excellent existing care/support at home) from admission to discharge; keen to demonstrate impact of SSPCI in AND patients; having named clinical lead nurse responsible for overall supervision of study proved beneficial and necessary; challenges talking to patients with long standing diagnoses about preferred place of care and future care planning. Patients reported feeling proud to take part; initial reluctance to attend hospice due to pre conceived ideas, but all now state look forward to coming. One participant decided participating was not for him and withdrew.

Conclusion Significant multiprofessional staff time and effort involved in delivering intervention - more than expected for a similar number of usual referrals. Staff recognised the vital importance of thorough initial clinical assessment on admission; used some new educational and inspirational outcome measures; further educated in whole 'research process' and realise importance of adhering to strict requirements; frustrated that unable to signpost patients elsewhere for support at end of interventions due to lack of available resources and/ or funding.

\section{P-82 BECOMING A RESEARCH ACTIVE HOSPICE: INTRODUCING RESEARCH INTO A DAY HOSPICE ENVIRONMENT}

Rebecca Grace Darge, Pauline Flanagan, Margaret Jarvis, Nicci Williamson, Claire Hookey. Douglas Macmillan Hospice, Stoke on Trent, UK

\subsection{6/bmjspcare-2017-hospice.109}

Background Despite the many perceived benefits of research in palliative care, hospices have historically not engaged with research to any significant degree (Payne et al., 2013). With the exception of small projects initiated by staff undertaking postgraduate studies, this hospice undertook no research until 2016. The Research Framework for Hospices was designed to start addressing this, encouraging Hospices to become Research Active.

Aims To become a Research Active hospice, with the knowledge, skills processes and organisational culture required to take part in research to commence recruitment to a multicentre trial within day hospice.

Methods A research governance framework was established, led by the hospice medical director with support from the Clinical Research Network (CRN) and neighbouring hospices who shared experience and policies. A study was identified from the National Institute for Health Research (NIHR) portfolio. It was decided to commence recruitment in day hospice. A number of hospice staff, including day hospice staff, undertook Good Clinical Practice training. Research awareness sessions were held, which included an introduction to the study. Results The hospice opened to recruitment in November 2016. By 25 May 2017, 95 day hospice patients have been screened, 45 were eligible and 26 have enrolled. Informal feedback has been positive, with staff appreciating the importance of research and being able to incorporate it within their clinical duties, and many patients being willing to take part.

Conclusions In under two years, the hospice has moved from a position where research was not 'something we do' to being actively involved in recruiting to a NIHR portfolio study. Key factors to success have included senior hospice leadership, the support of the CRN, and the involvement and enthusiasm of clinical staff. The next step is to widen involvement in research to other hospice clinical teams.

\section{P-83 THE JOURNEY BEGINS. TAKING THE FIRST STEPS INTO PORTFOLIO STUDIES. A HOSPICE PERSPECTIVE}

Toni Flanagan, Katie Taroni. St Giles Hospice, Lichfield, UK

\subsection{6/bmjspcare-2017-hospice.110}

Hospice UK (2013) acknowledged that research is not universally welcomed, encouraged or embedded within hospice care and highlighted the need for hospices to become more involved in research in the future. St Giles Hospice set out in 2014 to become a research active hospice. As a hospice that covers a wide geographical area providing a comprehensive service that includes two inpatient units, day hospice, specialist community team, hospice at home, family support and bereavement services, we are ideally placed to have access to hundreds of potential participants for clinical and non-clinical research studies.

How did St Giles Hospice become research active? A research operational policy was developed that set out a clear process for undertaking research within the hospice and led to the set up of a Research Forum which includes a research ethics committee. The research forum was set up with the following purpose: To develop and oversee the effective implementation of research at St Giles Hospice as part of the clinical governance process. To review all research proposals in accordance with the Operational Research Policy and Procedure. To ensure the protection of patients, carers, staff and volunteers through appropriate research governance processes which comply with the principles of The Research Governance Framework.

What St Giles Hospice has learnt from being part of portfolio studies: With the support from lead sites and the NIHR Clinical Research Network the hospice was able to learn the processes that are involved in conducting research. The organisational skills and time required to maintain records such as site files. The organisation was able to explore the research culture within the hospice. Patient engagement and appetite for research has been high. A better understanding about how high quality evidence is generated. 